\title{
Editorial
}

\section{Electrocardiography in Japan}

\author{
Eiichi KimURA, M.D.*
}

$\mathrm{T}^{\mathrm{s}}$

HE development of Japanese medicine can be roughly divided into

2 periods by the year of 1945 . During the period before 1945, it was mostly influenced by German medicine. German articles were read widely. After 1945, in the postwar period, American medicine has greatly affected the Japanese medicine; for example American ideas have come to be used for the classification of diseases.

In the field of electrocardiography, though we can see the similar tendency to some extent, it is not manifest as in general medicine. Even before 1945, the articles written in English were read more commonly than the German articles. This seems to be due to the historical fact that the main current of electrocardiography flowed from Einthoven to Lewis in England, and then from Lewis to Wilson in America. It is true that reports by Weber, Schütz, Samojloff and others, which were written in the German language, gave us considerable influences, but most of the Japanese electrocardiographists were more intrigued by the ideas of Lewis and Wilson which were more practical. As early as around 1940, studies based on doublet theory were made also in Japan, but these were not known in foreign countries because of the world war.

On the other hand, most electrophysiologists in Japan were influenced by German electrophysiology, which made them stick to what is called "Differenztheorie" (theory of algebraic summation) and made it difficult for them to understand the idea of Lewis and Wilson-the explanation of electrocardiograms by the theory of current distribution in the living body. Consequently, the efforts to make them understand the doublet theory had bcen continued by clinical electrocardiographists until the first half of the nineteen-fifties.

Some of the physiologists in Japan are still not satisfied by the doublet theory and are endeavoring to analyze the curves of the clinical electrocardiogram on basis of the action potential of individual muscle bundles. These studies are being made independently of the studies by Schaefer or

* Professor of Internal Medicine, Nippon Medical School, Tokyo. 
Robb.

However, the German school of electrocardiography had no little influence on Japanese clinical electrocardiography. The following may be a simple example. It is a classical fact pointed out by Wilson that wide $\mathrm{S}_{1}$ is a manifestation of right bundle branch block. In America this pattern is called simply right bundle branch block, while in European courtries this is called "Wilsonblock". In Japan this is generally called "Wilsonblock" after the terminology of German reports.

In Japan there is no special electrocardiographer. This comes from the difference in the hospital system. In Japan electrocardiograms are read by the internists who are interested in cardiology, especially in electrocardiography. In addition to their daily work as an electrocardiographer, they have to not only see in- and out-patients but educate students and interns in the field of general medicine. Such a system has advantage as well as shortcoming. Its shortcoming is that they cannot spare sufficient time for electrocardiographic diagnosis, for example the analysis of complicated arrhythmias. Its advantage is that they can read electrocardiograms with the knowledge of patient's general condition, which enables them to make a more reasonable diagnosis.

Although there is almost no difference between Japan and foreign countries in the diagnostic standards for electrocardiograms, difference does exist in the normal range of the amplitude of $\mathrm{R}$ deflection. Therefore, we cannot apply the American diagnostic criteria of cardiac hypertrophy to the Japanese. The Minnesota code made by Blackburn indicates that the upper limit of normal $R$ in $V_{5,6}$ is $26 \mathrm{~mm}$. If this is applied to the Japanese, false positive diagnosis of left ventricular hypertrophy becomes considerably frequent.

In other words, the normal defliction of $\mathrm{R}$ in the Japanese people is a little higher than in the Western people. This may be explained by the difference in bodily constitution between the Japanese and Western people. In the Japanese, the general physique, accordingly the size of of the thorax, is smaller and thickness of the subcutaneous tissue is also smaller than in the Western people, resulting in a closer localization of the heart to the chest wall. Consequently the electrical potential on the chest wall is high, frequently exceeding $26 \mathrm{~mm}$. even in the normal individuals. The majority of the cardiologists in Japan have agreed that the upper limit of $R$ in $V_{5,6}$ in the Japanese men should be $30 \mathrm{~mm}$. Similarly, the criteria of Sokolow and Lyon, in which $S v_{1}+R v_{5} \geqq 35 \mathrm{~mm}$. is considered to indicate the presence of left ventricular hypertrophy, should be corrected when used in the Japanese people and value of $40 \mathrm{~mm}$. is recognized to be more reasonable. Other values, such as time factors and 
criteria for $R S_{-} T$ deviation and for bundle branch block, require no such correction.

At present, 8,500 electrocardiographs are produced a year in Japan. Since there are about 100,000 doctors in Japan, this means that one electrocardiograph is produced for 12 doctors every year, though some of them are exported to foreign countries. In spite of the general acceptance that the incidence of cardiac disease in Japan is rather low compared with that in Western countries, the statistics of mortality reveals that cardiac diseases rank the third among the major causes of death in Japan. Moreover, in patients with cerebrovascular accidents, which is the most frequent cause of death in Japan, the electrocardiographic examination is also highly desirable. It may be said that the high incidence of these 2 conditions has led to the wide spread use of electrocardiograph in Japan. 